\title{
The Role of Apoptosis and Autophagy in the Hypothalamic-Pituitary-Adrenal (HPA) Axis After Traumatic Brain Injury (TBI)
}

\section{Serpil Taheri}

Erciyes Universitesi Tip Fakultesi

\section{Zuleyha Karaca}

Erciyes Universitesi Tip Fakultesi

Ecmel Mehmetbeyoglu

Cardiff University School of Medicine

\section{Zuhal Hamurcu}

Erciyes Universitesi Tip Fakultesi

\section{Zeynep Yılmaz}

Erciyes Universitesi Tip Fakultesi

\section{Fatma Dal}

Erciyes Üniversitesi: Erciyes Universitesi

\section{Venhar Cınar}

Erciyes Universitesi Tip Fakultesi

Halil Ulutabanca

Erciyes Universitesi Tip Fakultesi

\section{Fatih Tanriverdi}

Erciyes Universitesi Tip Fakultesi

Kursad Unluhizarci

Erciyes Universitesi Tip Fakultesi

\section{Minoo Rassoulzadegan}

Cote d'Azur Cancer Centre: Centre Azureen de Cancerologie

Fahrettin Kelestimur ( $\nabla$ fktimur@erciyes.edu.tr)

Yeditepe University School of Medicine: Yeditepe Universitesi Tip Fakultesi https://orcid.org/00000002-2861-4683

\section{Research Article}

Keywords: Traumatic brain injury (TBI), neuroinflammation, HPA axis, autophagy, apoptosis

Posted Date: February 1st, 2022 
DOI: https://doi.org/10.21203/rs.3.rs-1159219/v1

License: (a) (i) This work is licensed under a Creative Commons Attribution 4.0 International License. Read Full License 


\section{The Role of Apoptosis and Autophagy in the Hypothalamic-Pituitary-Adrenal (HPA) Axis after \\ 2 Traumatic Brain Injury (TBI)}

3 Serpil Taheri ${ }^{1,2}$, Zuleyha Karaca ${ }^{3}$, Ecmel Mehmetbeyoglu ${ }^{4}$, Zuhal Hamurcu ${ }^{1,2}$, Zeynep Y1lmaz ${ }^{1,2}$, Fatma Dal $^{2}$,

4 Venhar Cinar ${ }^{1,2}$, Halil Ulutabanca ${ }^{5}$, Fatih Tanriverdi ${ }^{3}$, Kursad Unluhizarci $^{3}$, Minoo Rassoulzadegan ${ }^{2,6}$, Fahrettin Kelestimur $^{7, *}$

5 Serpil Taheri, ORCID ID; 0000-0001-5806-8241, ${ }^{1}$ Department of Medical Biology, Erciyes University Medical School, Kayseri, Turkey, ${ }^{2}$ Betul-Ziya Eren

6 Genome and Stem Cell Center, Erciyes University, Kayseri, Turkey, staheri@erciyes.edu.tr,Tel: 0090352 2076666-13600, Fax: 0090-352 4379316

7 Zuleyha Karaca, ORCID ID; 0000-0003-3241-2352, ${ }^{3}$ Department of Endocrinology, Erciyes University Medical School, Kayseri, Turkey,

8 zuleyha@erciyes.edu.tr, Tel: 0090352 2076666-21913, Fax: 0090-352 4379316

9 Ecmel Mehmetbeyoglu, ORCID ID; 0000-0002-4597-6811, ${ }^{4}$ Department of Cancer and Genetics, Cardiff University, Cardiff, United Kingdom.

10 mehmetbeyogluse@ cardiff.ac.uk, Tel: 44 7495731490,

11 Zuhal Hamurcu, ORCID ID; 0000-0002-0711-4014, ${ }^{1}$ Department of Medical Bipology, Erciyes University Medical School, Kayseri, Turkey, ${ }^{2}$ Betul-Ziya

12 Eren Genome and Stem Cell Center, Erciyes University, Kayseri, zhamurcu@erciyes.edu.tr, Tel: 0090352 2076666-13600, Fax: 0090-352 4379316

13 Zeynep Yılmaz, ORCID ID; 0000-0002-1362-1717, ${ }^{2}$ Betul-Ziya Eren Genome and Stem Cell Center, Erciyes University, Kayseri, Turkey,

14 4021040020@erciyes.edu.tr, Tel: 0090352 2076666-13600, Fax: 0090-352 4379316

15 Fatma Dal, ORCID ID; 0000-0003-2035-3981, ${ }^{2}$ Betul-Ziya Eren Genome and Stem Cell Center, Erciyes University, Kayseri, Turkey,

16 fatmadal@erciyes.edu.tr Tel: 0090352 2076666-13600, Fax: 0090-352 4379316

17 Venhar Cinar, ORCID ID; 0000-0003-1544-8994, ${ }^{2}$ Betul-Ziya Eren Genome and Stem Cell Center, Erciyes University, Kayseri, Turkey,

18 venharcinar@erciyes.edu.tr, Tel: 0090352 2076666-13600, Fax: 0090-352 4379316

19 Halil Ulutabanca, ORCID ID; 0000-0001-5912-3222, ${ }^{5}$ Department of Neurosurgery, Erciyes University Medical School, Kayseri, Turkey,

20 ulutabanca@erciyes.edu.tr, Tel: 0090352 2076666-21707, Fax: 0090-352 4379316

21 Fatih Tanriverdi, ORCID ID; 0000-0001-8277-6774, ${ }^{3}$ Department of Endocrinology, Erciyes University Medical School, Kayseri, Turkey, 22 fatihtan@erciyes.edu.tr, Tel: 0090352 2076666-21914, Fax: 0090-352 4379316

23 Kursad Unluhizarci, ORCID ID;0000-0003-2024-7433, ${ }^{3}$ Department of Endocrinology, Erciyes University Medical School, Kayseri, Turkey,

24 kursad@erciyes.edu.tr, Tel: 009053357246 66, Fax: 0090-352 4379316

25 Minoo Rassoulzadegan ORCID ID; 0000-0002-5522-0647, ${ }^{2}$ Betul-Ziya Eren Genome and Stem Cell Center, Erciyes University, Kayseri, Turkey,

26 'Université Côte d'Azur, CNRS, Inserm, France, minoo.rassoulzadegan@unice.fr, Tel: 0033608324503 ,

27 Fahrettin Kelestimur ORCID ID; 0000-0002-2861-4683, ${ }^{7}$ Department of Endocrinology, Yeditepe University Medical School, Istanbul, TURKEY.

28 fktimur@erciyes.edu.tr, fahrettin.kelestemur@yeditepe.edu.tr, Tel: 009053392252 82, Fax: 0090 (0216) 5780575

29

30 Short Title: Apoptosis or autophagy in the HPA axis after TBI

\section{1 *Corresponding Author}

32 Fahrettin Kelestimur, M.D.

33 Department of Endocrinology

34 Yeditepe University Medical School

35 Istanbul, TURKEY.

36 E-mail: fktimur@erciyes.edu.tr

37 fahrettin.kelestemur@yeditepe.edu.tr 


\section{Abstract}

2 Traumatic brain injury (TBI) is one of the major health problems affecting millions of people worldwide leading to death or 3 permanent damage. TBI affects the hypothalamic-pituitary-adrenal (HPA) axis either by primary injury to the hypothalamic4 hypophyseal region or by secondary vascular damage, brain, and/or pituitary edema, vasospasm, and inflammation.

5 Neuroendocrine dysfunctions after TBI have been clinically described in all hypothalamic-pituitary axes. In this study, we

6 focused on the HPA axis-related tissues in the acute and chronic phases of TBI to determine the role of autophagy and apoptosis 7 in the development of endocrine disturbances after TBI.

8 We established a mild TBI (mTBI) in rats using the controlled cortical impact (CCI) model. The hypothalamus, pituitary, and 9 adrenal tissues were collected in the acute (24 hours) and chronic (30 days) groups after TBI; and we investigated autophagy $10(L c 3, B c \ln 1, P 150, U l k$, and Atg5) and apoptosis (pro-caspase-3, cleaved caspase-3) markers in the HPA axis-related tissues 11 using RT-PCR and Western blotting assays.

12 The present data showed that after mTBI, P150 and Atg 5 transcripts showed a stable decrement from the acute to chronic phases 13 in the hypothalamus, pituitary, and adrenal glands. In contrast, $B c \ln l$ transcripts were found to be only affected in the adrenal 14 glands, with a prominent increase during the chronic phase. We also evaluated the levels of TNF- $\alpha$ and corticosterone by western 15 blot in the HPA axis-related tissues in the acute and chronic phases after TBI. The increased corticosterone levels in the adrenal 16 glands pointed out the activation of the HPA axis, while high TNF- $\alpha$ levels in the hypothalamus, pituitary, and adrenal glands 17 suggested neuroinflammation.

18 As a result, transcripts related to autophagy were reduced in the hypothalamus, pituitary, and adrenals after TBI; but this was not 19 reflected in autophagy-related protein levels. In contrast, protein markers related to apoptosis increased in the adrenal glands 20 during the acute phase and in the pituitary during the chronic phase. TBI seems to trigger apoptosis by inducing inflammation 21 that can also affect the adrenals in the acute phase; and this situation may lead to an impairment of communication through the 22 hypothalamus, pituitary and adrenal glands. This may explain the permanent damage to the pituitary with increased apoptosis 23 and inflammation in the chronic phase. These findings may contribute to the elucidation of the underlying mechanisms of 24 endocrine dysfunctions such as pituitary and adrenal insufficiency that occur after TBI. Although adrenals are not directly 25 affected by TBI, we suggest that the role of the adrenals together with the hypothalamus and pituitary should not be ignored in 26 the acute phase after TBI.

27 Keywords: Traumatic brain injury (TBI), neuroinflammation, HPA axis, autophagy, apoptosis 


\section{Introduction}

2 Traumatic brain injury (TBI) is an important health problem worldwide that affects many people. Brain functions can be

3 temporarily or permanently impaired during TBI and a secondary injury may arise which is usually not detectable (Simon et al.

4 2017). The secondary injury could be associated with neuroinflammatory response and/or with a genetic predisposition

5 (Tanriverdi et al. 2008b). The hypothalamic-pituitary-adrenal (HPA) axis, which consists of the hypothalamus, pituitary, and

6 adrenal glands, is responsible for providing an adequate cortisol response to maintain the homeostasis of organisms in stressful

7 situations, such as in TBI (Karaca et al. 2021). TBI affects the HPA axis either by direct injury to the hypothalamic-hypophyseal

8 region or by secondary vascular damage, brain and/or pituitary edema, vasospasm, and inflammation (Özben 1998; Hiebert et

9 al. 2015; Mckee and Daneshvar 2015). Endocrine dysfunctions after TBI have been clinically described in all hypothalamic-

10 pituitary axes. Besides frequently seen neuropsychiatric symptoms including irritability, depression, and anxiety, one of the most

11 important neuroendocrine disorders emerging after TBI is pituitary insufficiency (Tanriverdi et al. 2015; Karaca et al. 2016).

12 Although these abnormalities that emerge after TBI are temporary in most patients, they can be permanent in some (Tanriverdi

13 et al. 2015).

14 The controlled cortical impact (CCI) animal model is mainly used to mimic human severe head trauma resulting in extensive

15 cortical loss, hippocampus and thalamic damage, cytotoxic and vasogenic brain edema, and increased intracranial pressure,

16 leading to focal damage. A significant objective of TBI models is to generate injuries with gradual modifications in morphology

17 and behavior, where the functional result is either enhanced or worsened by interference. The application of many TBI animal

18 models is critical for developing preventative measures and reducing the impact of various types of primary injury (Taylor et al.

19 2008).

20 The mechanism(s) of pituitary dysfunction, which can be seen in 27\% of the survivors (6-36 months) after moderate to severe

21 TBI, has/have not yet been fully elucidated (Tanriverdi et al. 2010). Although some neuroendocrine diseases associated with

22 abnormalities in the regulation of the HPA axis have been identified after TBI, it is not yet known which mechanisms play a role

23 in the emergence of these disorders in the HPA axis. The role of autophagy and apoptosis in the emergence of HPA axis

24 dysfunction after TBI is not known. It has been reported that TBI produced in a moderate CCI model, causes long-term

25 dysregulation of the neuroendocrine stress response (Taylor et al. 2008).

26 Secondary neuronal injury is linked to a neuroinflammatory response characterized by the generation of reactive oxygen species

27 (ROS) and inflammatory cytokines such as tumor necrosis factor (TNF), interleukin-1 (IL1), and interleukin-6 (IL6) after

28 primary head trauma. TNF- $\alpha$, in particular, is important in the neuroinflammation that occurs after TBI. TNF- $\alpha$ is a downstream 
1 inflammatory signaling pathway initiator, and its activation can start a cascade of inflammatory reactions(Tapp et al. 2019).

2 Many studies have shown increased secretion of TNF- $\alpha$, a neuroinflammatory marker from active astrocytes, and microglia in

3 the brain after TBI. Prolonged TNF- $\alpha$ production is harmful to neurons and leads to apoptosis and autophagy (Wolf et al. 2019;

4 Wu and Lipinski 2019).

5 Apoptosis is defined as a programmed cell death mediated by mitochondria in particular and results in nucleosomal DNA

6 fragmentation. There are 14 caspases known to be involved in the process of apoptosis in mammals. The initiator caspases

7 (caspases 8, 9, 10) are activated as a result of apoptotic stimulation, which leads to the activation of more effector caspases. It

8 has been shown that active cleaved caspase-3 is required to stimulate apoptosis in many studies.

9 Firstly, caspase-3 is synthesized as a 32-kilo Dalton (kDa) pro-enzyme, and then it is cleaved into 12 and $17 \mathrm{kDa}$ subunits. Two

10 subunits (12 k Da and two $17 \mathrm{k} \mathrm{Da}$ ) are reassembled to create the functionally active cleaved-caspase- 3 enzyme. After the

11 formation of the active cleaved caspase-3 enzyme, the effector caspases, which include caspase-3, -6 , and -7 , initiate the

12 degradation of many important cellular proteins. After the degradation of important proteins essential for the cell, finally,

13 chromatin condense and DNA is fragmented (Tan et al. 2017; Ye et al. 2017; Li et al. 2019).

14 Basal levels of autophagy provide the removal of abnormal proteins and aged or damaged organelles in the cell in the normal

15 state. However, when the autophagy mechanism is over-activated, it can trigger apoptosis. Autophagy is a very complicated

16 process regulated by the transcription of at minimum 15 genes and occurring at different well-coordinated stages, including

17 initiation, nucleation, elongation, and fusion with the lysosome. In these stages, varied autophagy-related (Atg) genes/proteins,

18 including Beclin-1 $(B \ln 1)$ and microtubule-associated light chain-3 play a major role and are frequently accepted as potential

19 markers of autophagic activity (Ozpolat and Benbrook 2015; Zhang and Wang 2018). Beclin-1 and LC3 proteins serve in

20 different stages of the autophagy mechanism. Beclin-1 is involved in the nucleation phase, the very early stage of autophagosome

21 formation, and is considered an essential component for the initiation of autophagy. LC3 protein has two forms, LC3-I and LC3-

22 II. LC3-I protein is localized in the cytoplasm under normal cell conditions. When the autophagy mechanism is triggered by

23 various stresses, such as hypoglycemia, hypoxia, and inflammation, a cytosolic form of LC3-I is transformed to LC3-II by

24 conjugation of a lipid molecule called phosphatidyl-ethanolamine (PE) for incorporation into the membrane of autophagosomes.

25 Therefore, LC3 is considered one of the important markers of autophagy (Ozpolat and Benbrook 2015; Klionsky et al. 2016;

26 Saha et al. 2018).

27 Although the role of apoptosis and autophagy have been investigated in different regions of the brain after TBI (Liu et al.

28 2008), evaluation of autophagy and apoptosis in the HPA axis after TBI may explain the mechanism of endocrine disturbances 
1 such as pituitary insufficiency after TBI. Therefore, in order to further characterize the effects of TBI on the HPA axis, the

2 present study was to investigate the markers of apoptosis and autophagy in the acute and chronic phases after mild-CCI to

3 reveal the underlying mechanism and the targeted glandes in the HPA axis dysfunction.

\section{2. Materials and Methods}

\section{2.1. Animals}

6 A total of 42 healthy 8-week-old adult male Sprague-Dawley rats weighing 250-300 g, were used in this study. These rats were

7 housed under a $12 \mathrm{~h} \mathrm{light/dark} \mathrm{cycle} \mathrm{at} 23^{\circ} \mathrm{C}$ and allowed ad libitum access to food and Erciyes University, GenKok, Transgenic

8 Department, Kayseri, Turkey. The animals were cared for and treated under the Laboratory Animal Care Principles (European

9 rules). All experiments were performed on randomized and controlled double-blind. All animal experimental protocols were

10 approved by The Local Ethics Committee for Animal Experiments of Erciyes University (No: 16/103).

\section{2.2. Models of TBI}

12 CCI techniques, parameters, and postoperative care have all been thoroughly reported previously (Bilgen 2005; Taylor et al.

13 2008). Rats were sedated with isoflurane (2.0-2.5 percent in $100 \% \mathrm{O} 2,2.0 \mathrm{~mL} / \mathrm{min}$ flow rate) and put in a stereotaxic frame with

14 the head in a horizontal plane about the interaural line. The spontaneous respiration of the rats was not suppressed during the

15 experiment. Depth of anesthesia was monitored by the chin and skeletal muscle tone. Every surgical procedure was carried out

16 in an aseptic environment. In this method, a craniotomy is performed, and head trauma is created in the area of interest with a

17 computerized pneumatic system at the desired speed, depth, and duration. After anesthesia, the scalp of the rat, which was fixed

18 in the prone position with a standard nailed frame, was shaved and the area was cleaned with $10 \%$ povidone-iodine, and the

19 periosteum was dissected by cutting the skin and fascia with a $10 \mathrm{~mm}$ median linear incision. The skin and galea were fixed to

20 the laterals with a bulldog clip to provide an adequate visual field. Then, in the left parasagittal region, a craniectomy defect was

21 produced by drilling the cranium with a $3 \mathrm{~mm}$ diameter dental drill $2 \mathrm{~mm}$ lateral to the sagittal suture and $2 \mathrm{~mm}$ posterior to the

22 bregma. By varying the velocity with which the exposed dura was struck and the depth of tissue compression, a mild injury was

23 induced (mild CCI: 20 psi, $1.5 \mathrm{~mm}$ ). Finally, the scalp was sutured with 3/0 silk and the wound was cleaned again with $10 \%$

24 povidone-iodine. No operation was performed on the Sham group. Following the injury, the animals were observed daily. Rats

25 that showed signs of pain (frozen, stooped posture, or vocalization) or infection (swelling, redness, or discharge) were eliminated

26 from the experiment, as were those that lost more than 20\% of their body weight (Bilgen 2005; Taylor et al. 2008). 


\section{2.3. Experimental Design}

2 As a result of the power analysis, the amount of Type I error (alpha) was 0.05 , the power of the test (1-beta) was 0.95 , and the

3 effect size was 0.82 while using the Independent Samples One-Way ANOVA test for minimum sample size required a significant

4 difference of 9 for each group; a total of 27 male rat was used. G-power (v3.1) was used for power analysis.

5 The male rats were randomly divided into three groups, and each group included 14 rats. The details were as follows: (1) control

6 group: sham- no operated group; (2) acute TBI: rats were sacrificed at 24 hours after TBI; (3) chronic TBI: rats were sacrificed

730 days after TBI. mRNA expression analyses were performed on 14 rats. Western blot analyses were performed on 9 rats

8 because enough protein was not obtained. All of the findings were derived from the entire total tissue of the hypothalamus,

9 pituitary and adrenals. Tissue samples were stored in Trizol (Thermo Fisher Scientific, USA) at $-80^{\circ} \mathrm{C}$ until use. The experimental

10 design is provided in Figure 1.

\section{2.4. Western Blot Analysis}

12 Standard procedures were used to extract total proteins from dissected hypothalamus, pituitary tissues, and adrenal glands. 13 (Hamurcu et al. 2016). A detergent-compatible protein assay kit was used to quantify the total protein concentration in each

14 sample (DC kit; Bio-Rad, Hercules, CA). For protein separation, aliquots comprising $40 \mathrm{~g}$ of total protein from each sample 15 were electrotransferred to polyvinylidene difluoride membranes using sodium dodecyl sulfate (SDS)-polyacrylamide gel 16 electrophoresis with a 4-20 percent gradient. The membranes were blocked with a blocking buffer $(0.1$ Triton X-100 in Tris17 buffered saline-Tween 20) [TBS-T] for 60 minutes. The membranes were probed with the primary antibodies listed following 18 after being washed with TBS-T: Tnf- $\alpha$ (Proteintech, Catalog no: 17590-1-AP), Corticosterone (MyBioSource, Catalog no: 19 MBS2006378), Lc3 (Cell Signaling, Catalog no:2775S), Beclin-1(Cell Signaling, Catalog no:3738S), pro-caspase-3 (Cell

20 Signaling, Catalog no:9662S), cleaved-caspase-3 (Cell Signaling, Catalog no:9661S), $\beta$-actin (Proteintech, Catalog no:60008-1-

$21 \mathrm{Ig}$ ). The membranes were treated with horseradish peroxidase-conjugated anti-rabbit (Bio-Rad, Catalog no:170-6515) or anti22 mouse secondary antibody (Bio-Rad, Catalog no:170-6516) after being rinsed with TBS-T. TBS-T containing 5\% dry milk was 23 used to dilute all antibodies. Clarity Western ECL Substrate (Bio-Rad) was used for chemiluminescence detection, and the blots 24 were viewed with a Chemidoc MP Imaging System (Bio-Rad) (Hamurcu et al. 2019). All antibodies were diluted in TBS-T 25 containing 5\% dry milk. The membranes were incubated with primary antibodies overnight at $4{ }^{\circ} \mathrm{C}$, then with secondary 26 antibodies for 1 hour. Chemiluminescence detection was performed with Clarity Western ECL Substrate (Bio-Rad) and the blots 27 were visualized with a Chemidoc MP Imaging System (Bio-Rad) and quantified with a densitometer using the imager application 
1 program (Bio-Rad Image Lab 5). All western blot experiments were independently repeated at least twice. All of the antibodies

2 were validated and made ready for use by the supplier company "according to the antibody validation principle of Uhlen et al.

3 (You can check manufactory web-site for antibody validation princible: https://www.cellsignal.com/about-us/cst-antibody-

4 validation-principles) (Bordeaux et al. 2010; Uhlen et al. 2016; Hamurcu et al. 2018, 2019; Pillai-Kastoori et al. 2020).

5

6 2.5. RNA Isolation and Real-Time PCR

7 Total RNA isolation was made from tissues using Trizol (Thermo Fisher Scientific, USA) and cDNA was synthesized from the

8 RNA samples using an Evo Script cDNA synthesis kit (Roche, Germany). The cDNA procedure was conducted according to the

9 manufacturer`s protocol and samples were quantified using a Roche 480 Real-Time PCR (Roche, Germany). Transcript levels

10 of Ulk, P150, Atg5 and Bcln1 genes in the hypothalamus-pituitary-adrenal of all groups were determined. Actb gene was used as

11 a housekeeping gene. All PCR experiments were repeated twice. The Ct values were normalized using the $2^{-\Lambda \Lambda C t}$ method (Livak

12 and Schmittgen 2001; Taheri et al. 2021).

\section{2.6. Statistical analysis}

14 The compliance of the data to normal distribution was evaluated by the histogram, q-q graphs, and Shapiro-Wilk test. For

15 determination of the differences in gene expression data, independent samples were analyzed by ordinary one-way ANOVA or

16 by Kruskal Walls test according to their distribution. If samples had normal distribution, parametric ordinary one-way ANOVA

17 test was used with Tukey post-hog test. If samples didn't have normal distribution non-parametric Kruskal Walls test with

18 Dunnett's post hog test was used.

19 Differences in protein expression were analyzed by unpaired student t-test or by Mann Whitney-U test according to their

20 distribution. If samples had normal distribution, parametric unpaired student t-test was used. If samples didn't have normal

21 distribution non-parametric Mann Whitney-U test was used

22 Graph-Pad Prism 8 packages were used for statistical analysis. Results with $\mathrm{p}$ values $<0.05$ were considered statistically

23 significant. All data are expressed as the mean with SD. The sample size was calculated with power analysis, the amount of Type

24 I error (alpha) was 0.05 , the power of the test (1-beta) was 0.95 , and the effect size was 0.82 while using the Independent Samples

25 One-Way ANOVA test for minimum sample size required a significant difference of 9 for each group; a total of 27 male rat was

26 used for G-power (v3.1) power analysis. 


\section{3. Results}

3 TBI was generated in rats using the controlled cortical impact (CCI) model. The hypothalamus, pituitary, and adrenal tissues

4 were removed in the acute (24 hours) and chronic phase (30 days).

5 The corticosterone levels were found to be increased in the adrenal tissue indicating HPA axis activation in the acute and chronic

6 phases of TBI. In addition, TNF- $\alpha$ protein levels were found to be increased in the hypothalamus, pituitary, and adrenal tissues

7 indicating neuroinflammation in the acute and chronic phases of TBI (Figure 2A-E). TNF- $\alpha$ protein was not detectable in the 8 hypothalamus of the sham group (Figure 2A, 2D).

9 Bcln1, P150, Ulk, and Atg5 mRNA and Lc3, Beclin-1, pro-caspase-3, cleaved caspase-3 (Clv-caspase-3) protein levels were 10 determined in the hypothalamus, pituitary and adrenal glands in the acute (24 hours) and chronic ( 30 days) phases after TBI.

\section{3.1. The decrease in the levels of transcripts initiated by TBI in the hypothalamus and pituitary from the acute phase}

\section{2 worsens during the chronic phase.}

13 Among the four transcripts tested, the $B c \ln 1$ transcript levels were slightly, albeit not significantly lower in the hypothalamus 14 and pituitary during the acute (respectively, $\mathrm{dF}=2,2 ; \mathrm{p}=0,41$ and $\mathrm{p}>0,99$ ) and chronic phases (respectively, $\mathrm{dF}=2,2 ; \mathrm{p}=0,73$ and $15 \mathrm{p}>0,15$ ) of TBI than the sham group (Figures 3A and 3B). The Ulk transcript levels were unchanged in the hypothalamus in the 16 acute $(\mathrm{dF}=2 ; \mathrm{p}=0,27)$ and chronic phases $(\mathrm{dF}=2 ; \mathrm{p}=0,43)$ of TBI (Figure $3 \mathrm{~A})$; but in the pituitary during the acute phase Ulk 17 transcripts were reduced $(\mathrm{dF}=2 ; \mathrm{p}=0,049)$, which was not maintained during the chronic phase $(\mathrm{dF}=2 ; \mathrm{p}>0,99)$ (Figure $3 \mathrm{~B})$. In 18 contrast, two other transcripts, $\mathrm{P} 150(\mathrm{dF}=2, \mathrm{p}=0.0004 ; \mathrm{dF}=2, \mathrm{p}<0.001)$ and $\operatorname{Atg} 5(\mathrm{dF}=2, \mathrm{p}=0.0012 ; \mathrm{dF}=2, \mathrm{p}=0.001)$ were

19 significantly decreased from the acute phase and further decreased in the chronic phase after TBI in the hypothalamus (Figure $203 \mathrm{~A}$ ) and pituitary compared to the sham group (Figure 3B).

\section{3.2. TBI induces distinct transcript profiles in the adrenals compared to the hypothalamus and pituitary from the acute}

\section{3 phase to the chronic phase.}

$24 B \ln 1$ was the only transcript to increase in the adrenal tissue of the chronic group compared to the acute group of TBI and the 25 sham group ( $\mathrm{dF}=2, \mathrm{p}=0.007)$ (Figure 3C). Three other transcript levels (Ulk, P150, and Atg5) were significantly decreased 26 compared to the sham group in the acute phase of TBI (respectively, $\mathrm{dF}=2, \mathrm{p}=0.011 ; \mathrm{dF}=2, \mathrm{p}=0.031 ; \mathrm{dF}=2, \mathrm{p}=0.023$ ) (Figure 27 3C). The levels of the Ulk and Atg5 transcripts were decreased compared to the sham group in the acute and chronic phases of 
1 TBI. The decrease was significant in the adrenal only in the acute phase (Ulk: $\mathrm{dF}=2, \mathrm{p}=0.025 ;$ Atg $5: \mathrm{dF}=2, \mathrm{p}=0.023$ ) (Figure $23 \mathrm{C})$.

3 3.3. TBI increases apoptosis in the adrenal and pituitary but the transcript levels of the autophagy markers are not 4 changed.

5 When autophagy is induced, cytosolic Lc3-I is transformed to Lc3-II and localized in autophagosome membranes by the addition

6 of PE. As a result, Lc3-II expression is widely considered to be a sign of autophagy induction (Lee et al. 2016). When autophagy

7 is induced, cytosolic Lc3-I is transformed to Lc3-II and localized in autophagosome membranes by the addition of PE. As a 8 result, Lc3-II expression is widely considered to be a sign of autophagy induction. Both Beclin-1 and Lc3 proteins are crucial 9 autophagy mediators and are involved in distinct phases of the autophagic process (Ueno et al. 2016). Western blot analysis 10 showed that Beclin-1 protein levels were similar in the hypothalamus, pituitary, and adrenal glands in the acute and chronic TBI 11 groups compared to the sham group (Figure 3D, Supplementary Figure 1).

12 To evaluate the occurrence of apoptosis after TBI in the hypothalamus, pituitary, and adrenal in the acute and chronic phases, 13 we assessed the expression of total caspase-3 and levels of Clv-caspase-3 (active form of caspase 3) using Western blot analysis. 14 There was no detectable level of Clv-caspase-3 protein in the hypothalamus in the acute and chronic phases after TBI (Figure 15 4A). We found that the induction of Clv-caspase-3 showed a marked increase in the acute phase in the adrenal and in the chronic 16 phase in the pituitary after TBI (Figure 4B, 4C).

\section{4. Discussion}

18 TBI leads to two types of injury, the primary and secondary injury which includes edema, disturbed blood flow, and

19 neuroinflammation, and has been implicated in the development of post-injury neuroendocrine diseases such as neuropsychiatric

20 disorders and pituitary insufficiency (Tanriverdi et al. 2010). The HPA axis is responsible for providing an adequate cortisol

21 response to maintain the homeostasis of organisms in stressful situations, such as TBI. Endocrine dysfunctions after TBI have

22 been clinically described in all hypothalamic-pituitary axes. In addition, hypopituitarism has been detected in $27 \%$ of the 23 survivors (6-36 months) after moderate to severe TBI. Although these abnormalities that emerge after TBI are temporary in most 24 patients, they are permanent in some (Tanriverdi et al. 2015).

25 Based on the evidence so far, it's tempting to speculate that chronic neuroinflammation plays a role in the development of long26 term endocrine dysfunctions following TBI, especially in people who have a hereditary susceptibility (Tanriverdi et al. 2008a, 27 b, 2010; Kasturi and Stein 2009). It is very important to investigate cellular mechanisms that may be beneficial for the 28 development of new treatments that can provide protection in secondary injury cases after TBI. 
1 We investigated whether autophagy or apoptosis was involved in the regulation of vital cell functions in the hypothalamus,

2 pituitary, and adrenal tissues of rats after the mild controlled cortical impact (CCI) model and we focused on the transcript and

3 protein markers associated with autophagy and apoptosis.

4 The HPA axis is activated acutely after a TBI as a result of the stress of the damage. Following the injury, TBI raises serum

5 corticosterone levels, which peak at 3 hours after TBI compared to controls and return to normal by 5 hours after TBI in rats.

6 This same increase in corticosterone levels together with the HPA axis activation is also seen in patients in the first 1-2 days

7 following mild and moderate TBI (Taylor et al. 2008). On the other hand, TBI may result in the HPA axis dysfunction

8 characterized by decreased corticosterone levels. Generally, corticosterone and TNF- $\alpha$ protein levels are measured from the sera

9 to detect the activation and neuroinflammation in the HPA axis (Taylor et al. 2008). In this study, we determined the

10 corticosterone and TNF- $\alpha$ protein levels in tissues-specifically by western blotting assay in order to show the effect of TBI only

11 in the target tissues. We have shown that the corticosterone levels, as a sign of the HPA axis activation in the adrenals, in the

12 acute and chronic phases after mild CCI model, increase as expected. In addition, increased TNF- $\alpha$ protein levels in the

13 hypothalamus, pituitary and adrenals in the acute and chronic phase after mild CCI were also evidence of increased

14 neuroinflammation.

15 Regarding autophagy transcripts, we demonstrated that the levels of $P 150$ and Atg 5 transcripts are significantly decreased during

16 the chronic phase in the hypothalamus and pituitary. In contrast, in the adrenals, the levels of P150 and Atg5 transcripts were

17 only decreased in the acute phase. The decrease in P150 and Atg5 protein levels in the hypothalamus is a sign of damage to the

18 neurons (Wu and Lipinski 2019). Here, we suggest that a decrease in P150 and Atg5 transcript levels in the adrenal tissue may

19 be associated with an increase in apoptosis ratio as well independently from autophagy.

20 We found that the levels of $U l k$ transcripts are decreased in the acute phase in the pituitary and adrenals after TBI. In the adrenal

21 tissue, in contrast to the hypothalamus and pituitary, the levels of $B c \ln 1$ transcripts were increased in the chronic phase.

22 Altogether, more robust alterations in the levels of transcripts were observed in the adrenal than in the hypothalamus and pituitary

23 in response to TBI. Previous studies have demonstrated that autophagy is activated post-TBI, and the investigators thought that

24 inhibition of the autophagic pathway might improve neurological deficits (Clark et al. 2008; Gao et al. 2017; Zhang and Wang

25 2018). However, none of these studies focused on the HPA axis-related tissues after TBI. According to our results, although the

26 transcript levels of autophagic markers showed significant changes in the hypothalamus, pituitary and adrenal in the acute and

27 chronic phase after TBI, these changes were not reflected in the autophagy related-protein levels of Lc3 and Beclin-1. In other

28 words, there was no change in the autophagic activity in the HPA axis-related tissues in the acute or chronic phase after TBI in 
1 terms of protein expression. Although the transcript levels of autophagic markers are significantly lower in all tissues after TBI,

2 especially during the chronic phase these changes were not reflected in the tissue protein levels of these transcripts. There appears

3 to be a role for autophagic transcripts in regulating the HPA axisafter TBI.

4 In the pituitary, the marker of apoptosis "Clv-caspase-3" expression was only detected in the chronic phase after TBI.

5 Remarkably, in the adrenal tissue, Clv-caspase-3 expression was increased in the acute phase, but it was not expressed in the

6 chronic phase. The transient increase in apoptosis during the acute phase after TBI in the adrenal may correspond to an active

7 regenerative process in the tissues. To date, no studies in the literature have investigated the response and situation of the adrenal

8 after TBI, therefore, more investigation is required on this issue which could provide information to prevent consequences from

9 secondary injury.

10 In 2011, Chen et al. created TBI in rats using the fluid percussion injury model and found that the rate of apoptosis increased in

11 the hypothalamus and pituitary in the 7th and 14th days after TBI (Chen et al. 2011). Tan et al. developed an intracranial

12 hypertension model to investigate its effects on the HPA axis caused by TBI. They found that 24-hour intracranial hypertension

13 increased the rate of apoptosis, particularly in the hypothalamus and pituitary (Tan et al. 2017). However, we found that the ratio

14 of apoptosis markers in the hypothalamus and pituitary did not change in the acute phase after TBI. In contrast, we found

15 increases in apoptosis marker Clv-Caspase-3 expression in the adrenal glands as the target organ in the acute phase of the HPA

16 axis.

17 Adrenal insufficiency due to suppressed activation of the HPA axis emerges in 11-13 percent of all TBI cases, according to

18 clinical evidence (Tanriverdi et al. 2015). After TBI, corticosterone (cortisol in human) levels are usually increased due to the

19 stress of the injury. On the other hand, TBI can also result in the HPA axis dysfunction characterized by decreased cortisol levels

20 (Taylor et al. 2008). The present study revealed the increased ratio of apoptosis in the acute phase of TBI in the adrenal glands,

21 in contrast, corticosterone levels were increased both in the acute and chronic phases after TBI (Garrahy and Agha 2016; Taheri

22 et al. 2021).

23 After TBI, although the literature has generally focused on the hypothalamus and pituitary (Tan et al. 2017; Zhang and Wang

24 2018; Wu and Lipinski 2019), we did not detect any changes in Clv-Caspase-3 protein expression in the hypothalamus in the

25 acute and chronic phases after TBI, which may be associated with differences in the model systems used. Many studies have

26 declared that apoptosis increases in the hypothalamus especially in the acute phase after TBI (Chen et al. 2011; Tan et al. 2017).

27 We found that the increase in Clv-caspase-3 protein expression was only detected in the acute phase in the adrenal tissue, and

28 subsequently only in the chronic phase in the pituitary. We speculated that neuroinflammation in the brain after TBI may trigger 
1 changes in the adrenal glands. In addition, an increased ratio of apoptosis in the adrenals in the acute phase may also disrupt

2 communication throughout the HPA axis and lead to permanent damage in the pituitary in the chronic phase. Finally, these

3 results may help to explain at least one of the underlying mechanisms of endocrine disturbances that occur after TBI, especially

4 the mechanism of pituitary insufficiency after TBI. Although adrenals are not directly affected by TBI, we suggest that the role

5 of the adrenal glands together with the hypothalamus and pituitary should not be ignored in the acute phase after TBI.

6

7 Determining which molecular mechanisms are playing a role in the HPA axis after TBI is crucial for both TBI treatment and 8 prevention of injuries (Tanriverdi et al. 2008a, 2010, 2015; Taheri et al. 2016). After TBI, HPA axis dysfunction can arise at

9 any level of the HPA axis and this situation may prevent the suppression of neuroinflammation by preventing the appropriate 10 response to stress.

12 Disclosure summary: The authors have nothing to disclose.

13 Funding Sources: This work was supported by the Erciyes University, Scientific Research Project Unit (Grant number 14 \# TOA-2017-6994).

\section{Author Contributions}

16 FK, KU, MR, ZK, FT, HU and ST conceived and designed the experiments and the primary research hypothesis; EM, ZY, FD 17 and ST created the TBI model and collected tissue from mice. ST, FD, VC and EM performed the experiments; EM and ZY 18 performed the statistical analysis and data representation; ZK, FT, HU and ST analyzed the draft manuscript and corrected the 19 style and content of the paper; and FK, KU, MR, ZK, HU and ST supervised all steps of this work, including the experiments. 20 All the aforementioned authors fully contributed to the reading, writing, and approval of the final version of this manuscript.

Bilgen M (2005) A new device for experimental modeling of central nervous system injuries. Neurorehabil Neural Repair

\section{References} 19:219-226. https://doi.org/10.1177/1545968305278635

Bordeaux J, Welsh AW, Agarwal

S, et al

(2010)

Antibody validation. Biotechniques 48:197-209. https://doi.org/10.2144/000113382

Chen X, Zhang B, Chai Y, et al (2011) Methylprednisolone exacerbates acute critical illness-related corticosteroid insufficiency associated with traumatic brain injury in rats. Brain Res 1382:298-307. https://doi.org/10.1016/j.brainres.2011.01.045 
human brain after trauma and critical illness. Autophagy 4:88-90. https://doi.org/10.4161/auto.5173

Gao Y, Zhuang Z, Gao S, et al (2017) Tetrahydrocurcumin reduces oxidative stress-induced apoptosis via the mitochondrial apoptotic pathway by modulating autophagy in rats after traumatic brain injury. Am J Transl Res 9:887-899

Garrahy A, Agha A (2016) How should we interrogate the hypothalamic-pituitary-adrenal axis in patients with suspected hypopituitarism? BMC Endocr Disord 16:1-8. https://doi.org/10.1186/s12902-016-0117-7

Hamurcu Z, Deliba N, Nalbantoglu U, et al (2019) FOXM1 plays a role in autophagy by transcriptionally regulating Beclin-1 and LC3 genes in human triple-negative breast cancer cells. 491-508

Hamurcu Z, Delibaşı N, Geçene S, et al (2018) Targeting LC3 and Beclin-1 autophagy genes suppresses proliferation, survival, migration and invasion by inhibition of Cyclin-D1 and uPAR/Integrin $\beta 1 /$ Src signaling in triple negative breast cancer cells. J Cancer Res Clin Oncol 144:415-430. https://doi.org/10.1007/s00432-017-2557-5

Hiebert JB, Shen Q, Thimmesch AR, Pierce JD (2015) Traumatic Brain Injury and Mitochondrial Dysfunction. Am J Med Sci 350:132-138. https://doi.org/10.1097/MAJ.0000000000000506

13 Karaca Z, Grossman A, Kelestimur F (2021) Investigation of the Hypothalamo-pituitary-adrenal (HPA) axis: a contemporary synthesis. Rev Endocr Metab Disord 22:179-204. https://doi.org/10.1007/s11154-020-09611-3

Karaca Z, Tanriverdi F, Ünlühizarci K, Kelestimur F (2016) GH and Pituitary Hormone Alterations after Traumatic Brain

Lee HY, Kim J, Quan W, et al (2016) Autophagy deficiency in myeloid cells increases susceptibility to obesity-induced

Li Z, Xu R, Zhu X, et al (2019) MicroRNA-23a-3p improves traumatic brain injury through modulating the neurological diabetes and experimental colitis. Autophagy 12:1390-1403. https://doi.org/10.1080/15548627.2016.1184799 apoptosis and inflammation response in mice. Cell Cycle 19:24-38. https://doi.org/10.1080/15384101.2019.1691763

Liu CL, Chen S, Dietrich D, Hu BR (2008) Changes in autophagy after traumatic brain injury. J Cereb Blood Flow Metab 28:674-683. https://doi.org/10.1038/sj.jcbfm.9600587 
method. Methods 25:402-408. https://doi.org/10.1006/meth.2001.1262

Mckee AC, Daneshvar DH (2015) The neuropathology of traumatic brain injury. Handb Clin Neurol 127:45-66. https://doi.org/10.1016/B978-0-444-52892-6.00004-0

Özben T (1998) Pathophysiology of Cerebral Ischemia. In: Free Radicals, Oxidative Stress, and Antioxidants. Springer US, Boston, MA, pp 163-187

Ozpolat B, Benbrook DM (2015) Targeting autophagy in cancer management-strategies and developments. Cancer Manag Res 7:291-299. https://doi.org/10.2147/CMAR.S34859

Pillai-Kastoori L, Heaton S, Shiflett SD, et al (2020) Antibody validation for Western blot: By the user, for the user. J Biol

Saha S, Panigrahi DP, Patil S, Bhutia SK (2018) Autophagy in health and disease: A comprehensive review. Biomed Pharmacother 104:485-495. https://doi.org/10.1016/j.biopha.2018.05.007

Simon DW, McGeachy M, Bayir H, et al (2017) Neuroinflammation in the Evolution of Secondary Injury, Repair, and Chronic Neurodegeneration after Traumatic Brain Injury. Nat Rev Neurol 13:171-191. https://doi.org/10.1038/nrneurol.2017.13.Neuroinflammation

Taheri S, Karaca Z, Rassoulzadegan M, et al (2021) The Characterization of Sex Differences in Hypoglycemia-Induced Activation of HPA Axis on the Transcriptomic Level. Cell Mol Neurobiol. https://doi.org/10.1007/s10571-021-01043-0

Taheri S, Tanriverdi F, Zararsiz G, et al (2016) Circulating MicroRNAs as Potential Biomarkers for Traumatic Brain InjuryInduced Hypopituitarism. J Neurotrauma 33:1818-1825. https://doi.org/10.1089/neu.2015.4281

Tan H, Yang W, Wu C, et al (2017) Assessment of the role of intracranial hypertension and stress on hippocampal cell apoptosis and hypothalamic-pituitary dysfunction after TBI. Sci Rep 7:1-12. https://doi.org/10.1038/s41598-017-04008-w

Tanriverdi F, De Bellis A, Bizzarro A, et al (2008a) Antipituitary antibodies after traumatic brain injury: Is head traumainduced pituitary dysfunction associated with autoimmunity? Eur J Endocrinol 159:7-13. https://doi.org/10.1530/EJE-

Tanriverdi F, Schneider HJ, Aimaretti G, et al (2015) Pituitary dysfunction after traumatic brain injury: A clinical and 08-0050

Tanriverdi F, Taheri S, Ulutabanca H, et al (2008b) Apolipoprotein E3/E3 genotype decreases the risk of pituitary dysfunction pathophysiological approach. Endocr Rev 36:305-342. https://doi.org/10.1210/er.2014-1065 after traumatic brain injury due to various causes: Preliminary data. J Neurotrauma 25:1071-1077. https://doi.org/10.1089/neu.2007.0456 
1 Tanriverdi F, Unluhizarci K, Kelestrimur F (2010) Persistent neuroinflammation may be involved in the pathogenesis of traumatic brain injury (TBI)-induced hypopituitarism: Potential genetic and autoimmune factors. J Neurotrauma 27:301302. https://doi.org/10.1089/neu.2009.1102

Tapp ZM, Godbout JP, Kokiko-Cochran ON (2019) A Tilted Axis: Maladaptive Inflammation and HPA Axis Dysfunction Contribute to Consequences of TBI. Front Neurol 10:1-19. https://doi.org/10.3389/fneur.2019.00345

Taylor AN, Rahman SU, Sanders NC, et al (2008) Injury severity differentially affects short- and long-term neuroendocrine outcomes of traumatic brain injury. J Neurotrauma 25:311-323. https://doi.org/10.1089/neu.2007.0486

Ueno T, Saji S, Sugimoto M, et al (2016) Clinical significance of the expression of autophagy-associated marker, beclin 1, in breast cancer patients who received neoadjuvant endocrine therapy. BMC Cancer 16:1-9. https://doi.org/10.1186/s12885-016-2270-9

Uhlen M, Bandrowski A, Carr S, et al (2016) A proposal for validation of antibodies. Nat Methods 13:823-827. https://doi.org/10.1038/nmeth.3995

13 Wolf MS, Bayır H, Kochanek PM, Clark RSB (2019) The role of autophagy in acute brain injury: A state of flux? Neurobiol Dis 122:9-15. https://doi.org/10.1016/j.nbd.2018.04.018 https://doi.org/10.3390/cells8070693

Ye Y, Zhang P, Qian Y, et al (2017) The Effect of Pyrroloquinoline Quinone on the Expression of WISP1 in Traumatic Brain Injury. Stem Cells Int 2017:. https://doi.org/10.1155/2017/4782820 


\section{Graphical Abstract}

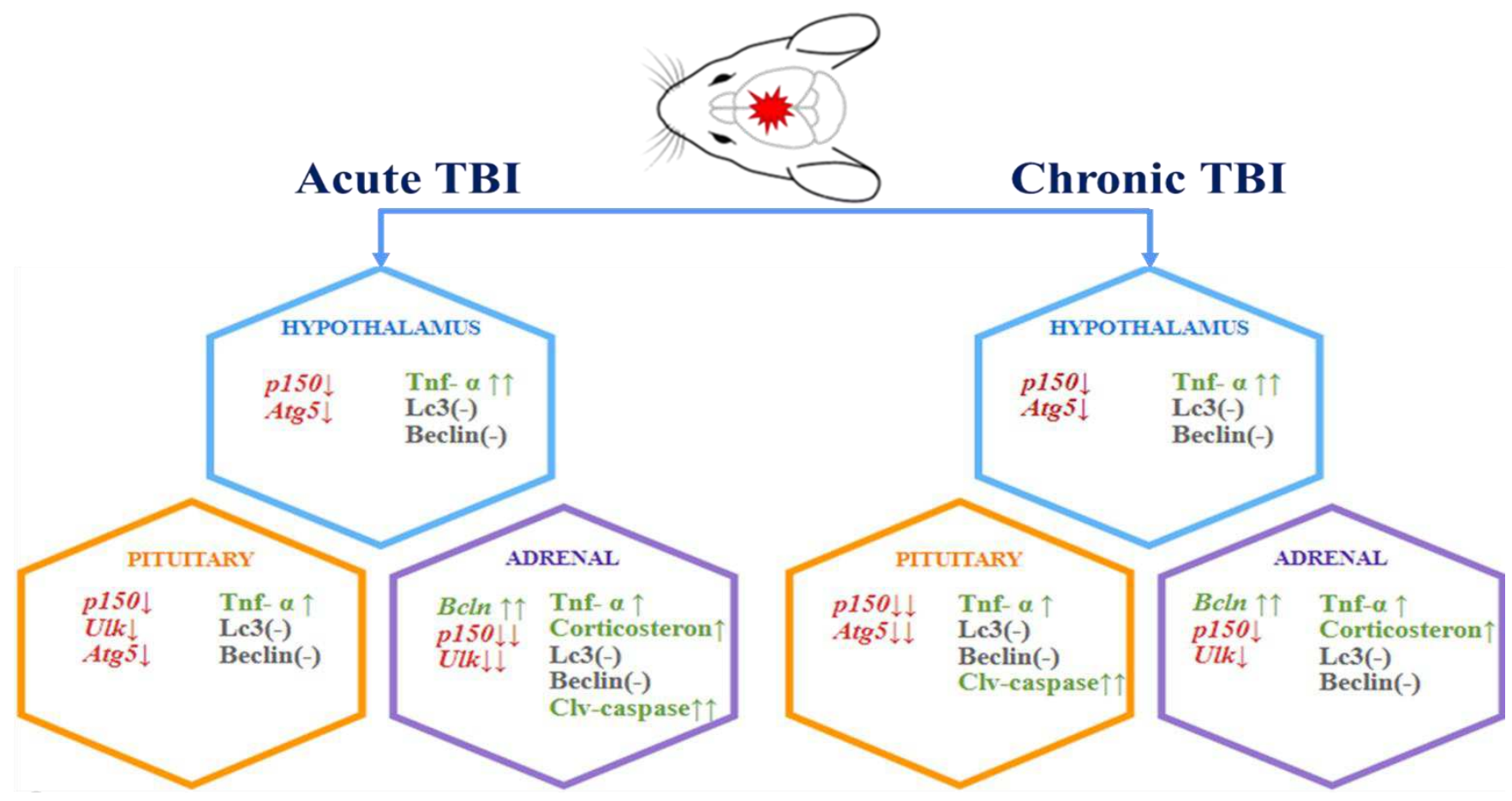




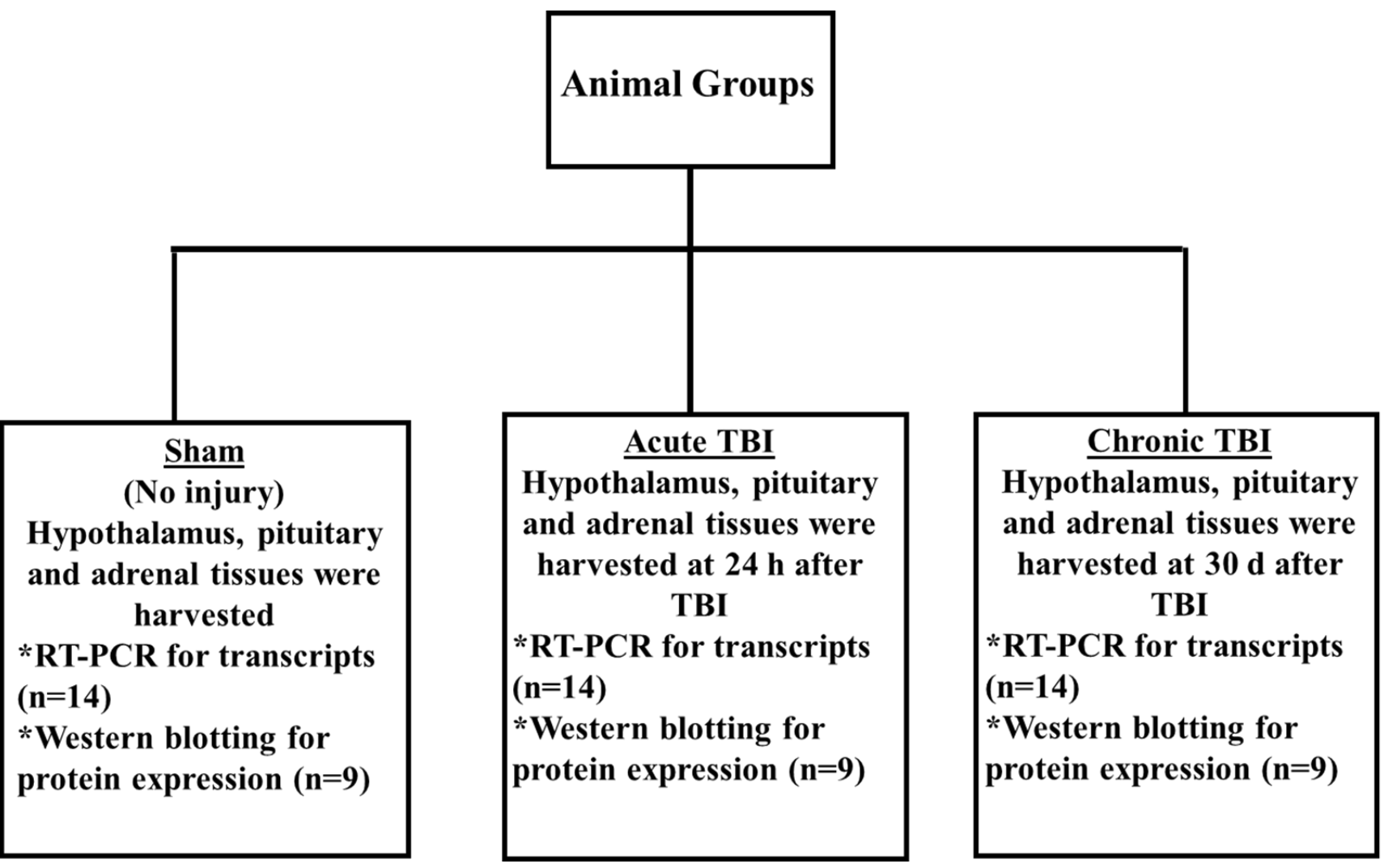

2 Fig. 1 Experimental Design. Anesthetized male rats were subjected to TBI with the The controlled cortical

3 impact $(\mathrm{CCI})$ or sham procedure (without $\mathrm{CCI}$ ). Sham animals were sacrificed directly without any

4 operation. Acute TBI group was sacrificed at $24 \mathrm{~h}$ after TBI with CCI. Chronic TBI group animals were

5 sacrificed at 30 days after TBI with CCI. The male rats were randomly divided into three groups, and each

6 group included 14 rats. The details were as follows: (1) control group: sham- no operated group; (2) acute

7 TBI: rats were sacrificed at 24 hours after TBI; (3) chronic TBI: rats were sacrificed 30 days after TBI.

8 Gene expression analyses were performed on 14 rats and western blot analyses on 9 rats. Biological and

9 technical replicates for each experiment were added included in both the manuscript and figure legends

10 as below. Gene expression analyses were performed on 14 rats and western blot analyses on 9 rats. All

11 PCR experiments and western blot analyses for detecting transcript and protein levels were repeated at

12 least twice independently and blindly.

13 
A

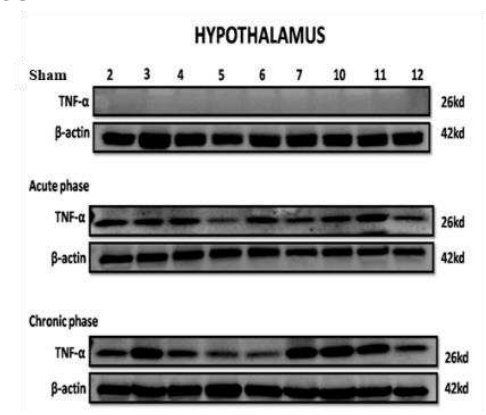

B
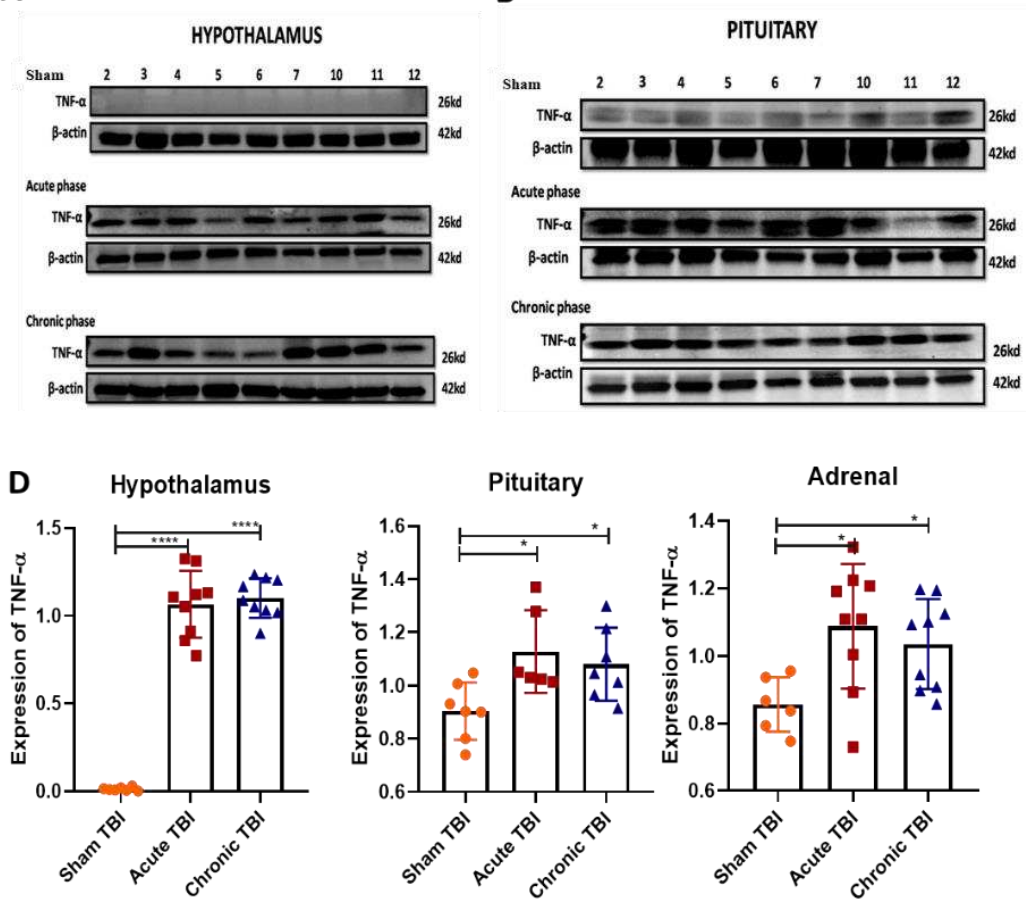

C

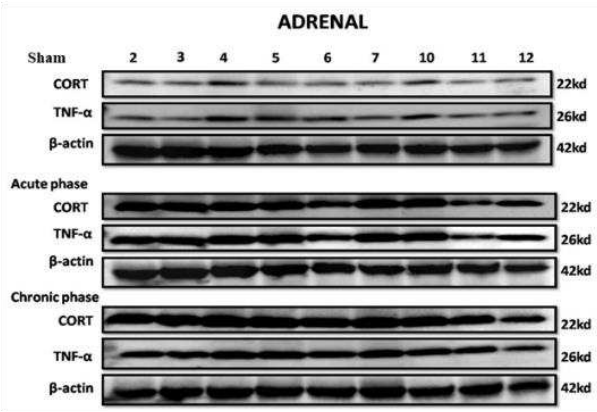

E Adrenal Cort

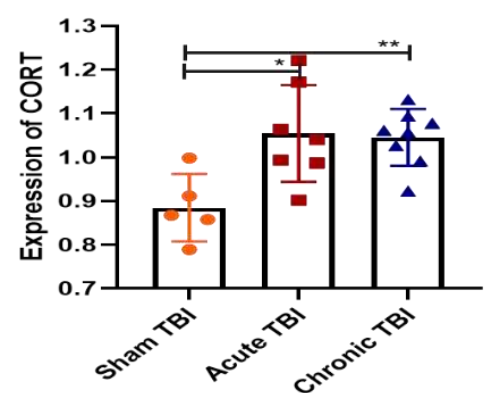

Fig. 2

2 Fig. 2 Western blot images of Tnf- $\alpha$ and Corticosterone (Cort) levels in the acute and chronic phase after

3 TBI A. Tnf- $\alpha$ protein levels in the hypothalamus in the acute and chronic phases after TBI. $\beta$-Actin was

4 used as a loading control. Data are presented as mean $\pm \mathrm{SD}(\mathrm{n}=9$ rats in each group). B. Tnf- $\alpha$ protein

5 levels in the pituitary in the acute and chronic phases after TBI. C.Tnf- $\alpha$ and Cort levels in the adrenal in

6 the acute and chronic phases after TBI. $\beta$-Actin was used as a loading control. Data are presented as mean

$7 \pm \mathrm{SD}(\mathrm{n}=9$ rats in each group). D. The graph showing Tnf- $\alpha$ protein levels in the hypothalamus, pituitary,

8 and adrenal in the acute and chronic phases after TBI. $\beta$-Actin was used as a loading control. Data are

9 presented as mean $\pm \mathrm{SD}(\mathrm{n}=9$ rats in each group). $(* \mathrm{p}<0.05, * * \mathrm{p}<0.001, * * * * \mathrm{p}<0.0001)$. All western

10 blot analyses were independently repeated at least twice.

11

12

13 

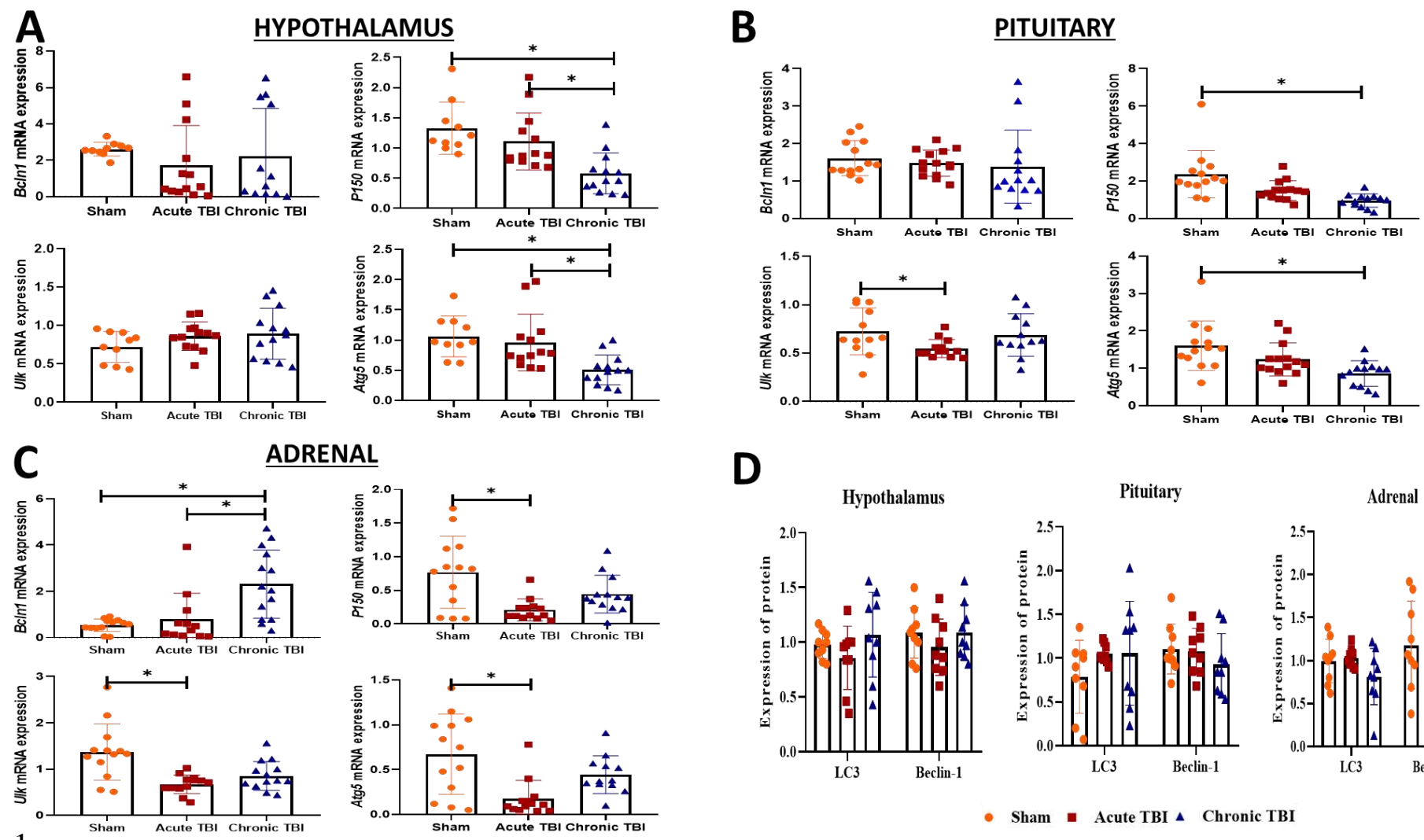

D

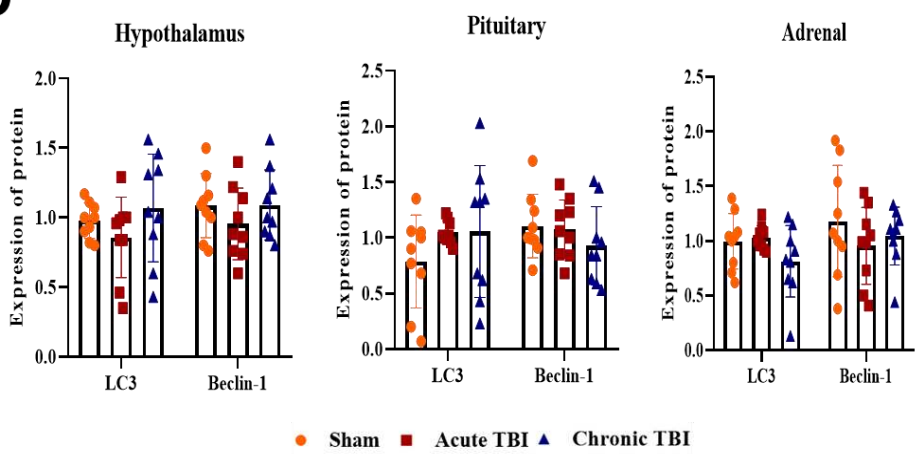

2 Fig 3. A. The transcript levels of $B c \ln 1, P 150$, Ulk and $\operatorname{Atg} 5$ genes in the hypothalamus after TBI (A, B,

3 C and D) (Bar Graph) $\left({ }^{*} \mathrm{p}<0.05\right)$. Box plots are expressed as mean $\pm \mathrm{SD}(\mathrm{n}=14$ rats in each group). B.

4 The transcript levels of $B c \ln 1, P 150$, Ulk and $A \operatorname{tg} 5$ genes in the pituitary after TBI (A, B, C and D) (Bar

5 Graph) $\left({ }^{*} \mathrm{p}<0.05\right)$. Box plots are as mean $\pm \mathrm{SD}(\mathrm{n}=14$ rats in each group). C. The transcript levels of

6 Bcln 1, P150, Ulk and Atg5 genes in the adrenal after TBI (A, B, C and D) (Bar Graph) $\left({ }^{*} \mathrm{p}<0.05\right)$. Box

7 plots are expressed as mean $\pm \mathrm{SD}$ ( $\mathrm{n}=14$ rats in each group). All PCR experiments for detecting transcript

8 levels were repeated at least twice independently and blindly. D. The graph showing LC-3 and Beclin-1

9 protein levels in the hypothalamus, pituitary, and adrenal in the acute and chronic phases after TBI. Data

10 are presented as mean $\pm \mathrm{SD}(\mathrm{n}=9$ rats in each group). All western blot analyses were independently

11 repeated at least twice 
A

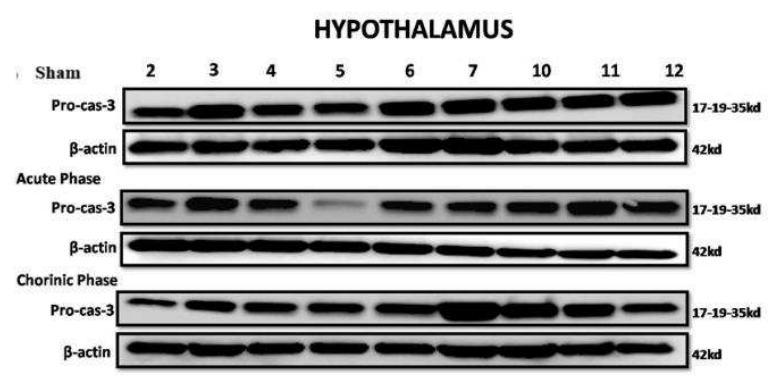

B

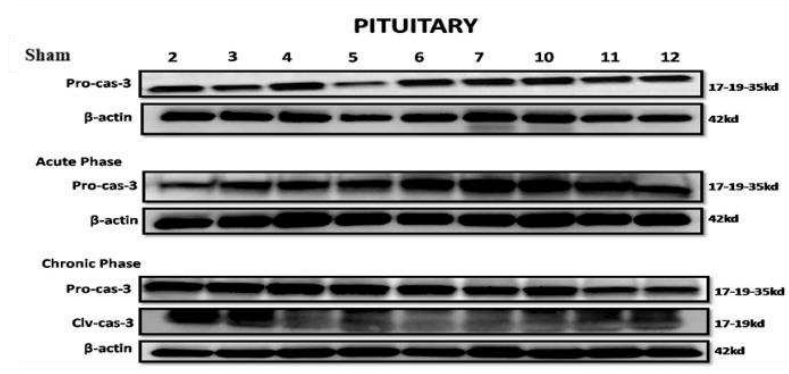

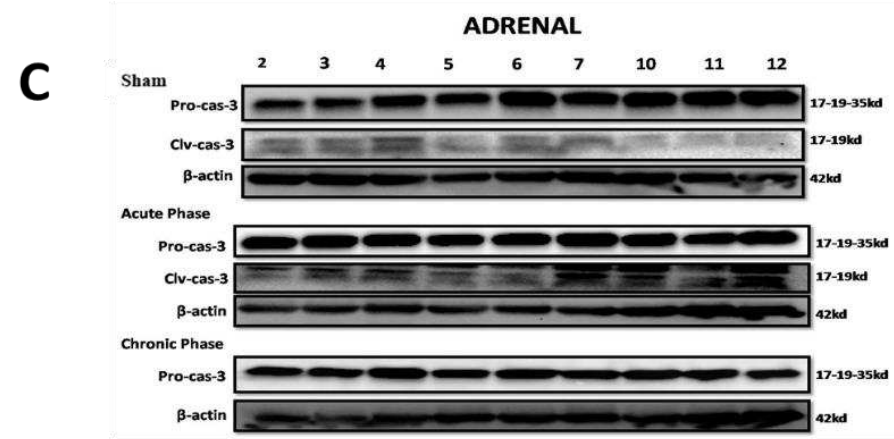

2 Fig. 4 Western blot images of Clv-caspase-3 in the acute and chronic phase after TBI in the hypothalamus,

3 pituitary, and adrenal A. Clv-caspase-3 protein levels in the hypothalamus in the acute and chronic phases

4 after TBI. B.Clv-caspase-3 protein levels in the pituitary in the acute and chronic phases after TBI. C.Clv-

5 caspase-3 protein levels in the adrenal in the acute and chronic phases after TBI. $\beta$-Actin was used as a

6 loading control. Data are presented as mean \pm SD $(n=9$ rats in each group). All western blot analyses

7 were independently repeated at least twice. 


\section{Supplementary Files}

This is a list of supplementary files associated with this preprint. Click to download.

- supplementfigure2.pptx 